\title{
Symptomatic hypophosphataemia after intravenous iron therapy: an underrated adverse reaction
}

\author{
Eseoghene Ifie', Samson O Oyibo², Hareesh Joshi² and Olugbenro O Akintade ${ }^{3}$ \\ ${ }^{1}$ Nuffield Department of Clinical Neurosciences, John Radcliffe Hospital, Oxford, UK, 2Department of Endocrinology, \\ and ${ }^{3}$ Department of Elderly Care Medicine, Peterborough City Hospital, Peterborough, UK
}

Correspondence should be addressed to $S$ O Oyibo

Email

samoyibo@yahoo.co.uk

\section{Summary}

Iron (ferric carboxymaltose) infusion therapy is used to treat severe iron deficiency which is not responding to the first-line oral iron therapy. However, it can also cause severe renal wasting of phosphate resulting in severe hypophosphataemia in some patients. Despite the growing number of case reports, this side effect is not well known to healthcare professionals. The product labelling information sheet does mention that hypophosphataemia can be a side effect, but also says that this side effect is usually transient and asymptomatic. We report a challenging case of a patient who developed severe, symptomatic and prolonged hypophosphataemia after an intravenous iron infusion for severe iron deficiency.

\section{Learning points:}

- Clinicians prescribing ferric carboxymaltose (Ferinject ${ }^{\circledR}$ ) should be aware of the common side effect of hypophosphataemia, which could be mild, moderate or severe.

- Patients receiving iron infusion should be educated concerning this potential side effect.

- Pre-existing vitamin D deficiency, low calcium levels, low phosphate levels or raised parathyroid hormone levels may be risk factors, and these should be evaluated and corrected before administering intravenous iron.

- Patients may require phosphate and vitamin D replacement along with monitoring for a long period after iron infusion-induced hypophosphataemia.

- Every incident should be reported to the designated body so that the true prevalence and management thereof can be ascertained.

\section{Background}

Iron deficiency is an important cause of anaemia affecting patients with chronic kidney disease, inflammatory bowel disease, intestinal malabsorption syndromes and women who have heavy menstrual bleeding. Not all cases are associated with anaemia. In the absence of anaemia, iron deficiency still produces symptoms such as fatigue, irritability, apathy and depression. Therefore, iron replacement therapy is usually commenced, while the patient is undergoing investigations for the underlying cause.

Oral iron supplementation is the usual first-line therapy for iron deficiency, but this has a high rate of gastrointestinal side effects, low bioavailability and nonadherence to treatment is common (1). For this reason, the parenteral route was developed and is now the preferred route, as this allows the administration of large amounts of iron $(1000 \mathrm{mg})$ in one single infusion (1).

The product labelling information sheet for the iron infusion (ferric carboxymaltose) does mention that it can cause hypophosphataemia and that it is usually transient and asymptomatic. This side effect is also mentioned under common or very common in the British National Formulary. However, this is not 
widely acknowledged by healthcare professionals who prescribe this iron product.

Ferric carboxymaltose infusion therapy causes renal wasting of phosphate. It has been suggested that this iron compound increases serum levels of fibroblast growth factor 23 (FGF23) by inhibiting FGF23 degradation. FGF23 is a peptide secreted by both osteoclasts and osteoblasts in response to raised phosphate levels. Its function is to limit phosphate reabsorption in the renal tubules, maintaining phosphate homeostasis. Therefore, the resultant excess serum FGF23 causes hypophosphataemia by inappropriately decreasing phosphate reabsorption in the proximal renal tubules even in the presence of continued low serum phosphate levels. Additionally, FGF23 has been reported to inhibit the production of calcitriol which is needed for phosphate absorption in the intestines (2).

We report a challenging case of a patient who developed severe, symptomatic and prolonged hypophosphataemia after an intravenous iron infusion.

\section{Case presentation}

A 43-year-old Caucasian lady presented to the emergency department with a 3-day history of palpitations and chest discomfort. There was a past medical history of chronic iron deficiency secondary to heavy uterine bleeding being treated with oral iron tablets (ferrous sulphate). She had poor adherence to taking the iron tablets because of gastrointestinal side effects and the fact that she did not like taking tablets. Serum ferritin levels had been below $30 \mu \mathrm{g} / \mathrm{L}$ for the past 2 years. As a result of non-response to prolonged oral iron supplementation and a serum ferritin level still low at $17 \mu \mathrm{g} / \mathrm{L}$, the patient received an intravenous infusion of ferric carboxymaltose (Ferinject ${ }^{\circledR}$ ) 6 days prior to this presentation. The patient was scheduled to receive a $1000 \mathrm{mg}$ dose made up to $270 \mathrm{~mL}$ with $0.9 \%$ sodium chloride solution to run over $30 \mathrm{~min}$, but tolerated only half of the infusion, because of a severe anxiety attack. Examination revealed no abnormal features. Her electrocardiogram was normal. Initial blood results were normal apart from the serum phosphate level being slightly low at $0.6 \mathrm{mmol} / \mathrm{L}$ (normal range: $0.8-1.5 \mathrm{mmol} / \mathrm{L})$. She was reassured and sent home with effervescent phosphate tablets (Sandoz Phosphate ${ }^{\circledR}$ ).

The patient presented again 3 days later with symptoms of painful cramps in both legs, dizziness, fatigue and nausea and was found to have a serum phosphate level of $0.43 \mathrm{mmol} / \mathrm{L}$. The patient could not tolerate the effervescent phosphate tablet because it caused severe nausea and painful indigestion. There was no past history of undernutrition or intake of any medications that would interfere with phosphate metabolism.

\section{Investigation}

Other blood results were normal, and the serum ferritin level was appropriately raised in response to the recent intravenous iron administration (Table 1). Urinalysis demonstrated a fractional excretion of phosphate $\left(\mathrm{FEPO}_{4}\right)$ of $24.2 \%$, which confirmed severe renal wasting of phosphate in the presence of severe hypophosphataemia $\left(\mathrm{FEPO}_{4}\right.$ should be less than $5 \%$ in the presence of hypophosphataemia). Urinalysis for aminoaciduria ruled out underlying renal tubular disorders. Blood tests done 4 months prior to the iron infusion revealed normal serum creatinine, calcium, phosphate $(0.94 \mathrm{mmol} / \mathrm{L})$ and vitamin D levels. This ruled out any form of underlying or pre-existing hypophosphataemia or problems with phosphate and calcium metabolism. Moreover, FGF23 levels were raised at $225 \mathrm{RU} / \mathrm{ml}$ (normal $<100 \mathrm{RU} / \mathrm{ml}$ ).

\section{Treatment}

The patient was admitted and given an intravenous infusion of Phosphate Polyfusor ${ }^{\circledR}, 150 \mathrm{~mL}$ infused over

Table 1 Haematology and biochemistry results (with normal values) prior to the iron infusion and at presentation.

\begin{tabular}{|c|c|c|c|}
\hline \multirow[b]{2}{*}{$\begin{array}{l}\text { Blood parameters } \\
\text { (units) }\end{array}$} & \multirow[b]{2}{*}{$\begin{array}{c}\text { Normal } \\
\text { ranges }\end{array}$} & \multicolumn{2}{|c|}{ Patient's results } \\
\hline & & $\begin{array}{l}4 \text { months } \\
\text { prior to iron } \\
\text { infusion } \\
\end{array}$ & $\begin{array}{c}\text { At presentation } \\
\text { (after iron } \\
\text { infusion) } \\
\end{array}$ \\
\hline Haemoglobin (g/L) & $115-165$ & 153 & 140 \\
\hline White blood cells & $4.0-11.0$ & 8.8 & 7.0 \\
\hline Platelets (109/L) & $150-400$ & 265 & 242 \\
\hline Sodium (mmol/L) & $132-145$ & 141 & 142 \\
\hline Potassium (mmol/L) & $3.4-5.1$ & 4.5 & 4.4 \\
\hline Creatinine $(\mu \mathrm{mol} / \mathrm{L})$ & $45-84$ & 78 & 67 \\
\hline $\begin{array}{l}\text { Adjusted calcium } \\
(\mathrm{mmol} / \mathrm{L})\end{array}$ & $2.20-2.60$ & 2.27 & 2.35 \\
\hline Phosphate (mmol/L) & $0.80-1.50$ & 0.94 & 0.43 \\
\hline $\begin{array}{l}\text { 25-hydroxy vitamin D } \\
\text { (nmol/L) }\end{array}$ & $>50$ & 76 & 59 \\
\hline Magnesium (mmol/L) & $0.7-1.0$ & - & 0.74 \\
\hline $\begin{array}{l}\text { Parathyroid hormone } \\
\text { (pmol/L) }\end{array}$ & $1.4-6.2$ & - & 6.0 \\
\hline $\begin{array}{l}\text { Alkaline Phosphatase } \\
(U / L)\end{array}$ & $30-130$ & 88 & 89 \\
\hline Ferritin $(\mu \mathrm{g} / \mathrm{L})$ & $30-400$ & 13 & 481 \\
\hline
\end{tabular}

Serum ferritin level was $17 \mu \mathrm{g} / \mathrm{L}$ a month prior to the iron infusion. Serum ferritin levels went back down to $100 \mu \mathrm{g} / \mathrm{L}$ on day 19 (within 3 weeks of recent iron infusion). PTH was measured using the Elecsys ${ }^{\circledR}$ PTH assay, which measures intact PTH and has monoclonal antibodies against both the $\mathrm{N}$-terminal and C-terminal regions. 
$12 \mathrm{~h}$ (containing $15 \mathrm{mmol}$ of phosphate). The patient could not tolerate the effervescent phosphate tablets, which gave her severe chest and epigastric discomfort. Because of recurrent low serum phosphate levels and symptoms the patient required 15 doses of intravenous Phosphate Polyfusor ${ }^{\circledR}$ over a 4-week hospital stay period. The patient was also given calcitriol $0.25 \mu \mathrm{g}$ daily, vitamin D3 800 units daily, a phosphate-rich diet and a bespoke syrup phosphate (sodium dihydrogen phosphate $15 \mathrm{mmol}$ three times a day) which the patient was just about able to tolerate. The calcitriol dose was later increased to $0.25 \mu \mathrm{g}$ twice a day. The patient's serum calcium, magnesium, phosphate and creatinine levels were monitored throughout the treatment period.

The last phosphate infusion was administered on day 26 and the patient was discharged on day 30 on a regimen of calcitriol $0.25 \mu \mathrm{g}$ twice a day, vitamin D3 800 units daily, syrup sodium dihydrogen phosphate $15 \mathrm{mmol}$ three times a day, and some effervescent phosphate tablets. The serum phosphate levels are shown in Fig. 1.

\section{Outcome and follow-up}

The patient's serum phosphate levels remained in the normal range while on a reducing dose of phosphate supplements, during outpatient monitoring (Fig. 1). A repeat serum ferritin level was much lower $(67 \mu \mathrm{g} / \mathrm{L})$ and the patient was advised to recommence oral iron tablets. On the 42nd day, the patient was able to stop taking phosphate supplements altogether and the patient's serum phosphate levels remained in the normal range thereafter.

\section{Discussion}

We have reported a case of symptomatic hypophosphataemia occurring within a week after the patient received $519 \mathrm{mg}$ of intravenous ferric carboxymaltose (Ferinject ${ }^{\circledR}$ ) for severe iron deficiency. The very high $\mathrm{FEPO}_{4}$ indicated that the hypophosphataemia resulted from significant renal phosphate wasting, secondary to the iron infusion. Renal function, phosphate and calcium metabolism were normal prior to the iron infusion. Unfortunately, the patient could not tolerate oral phosphate supplements so required frequent intravenous phosphate replacement.

Hypophosphataemia secondary to intravenous iron (notably ferric carboxymaltose) has been reported, but this has been generally thought to be both asymptomatic and transient. However, recent reports have noted that this side effect is not as asymptomatic and transient as previously

\section{Serum phosphate levels ( $\mathrm{mmol} / \mathrm{L})$}

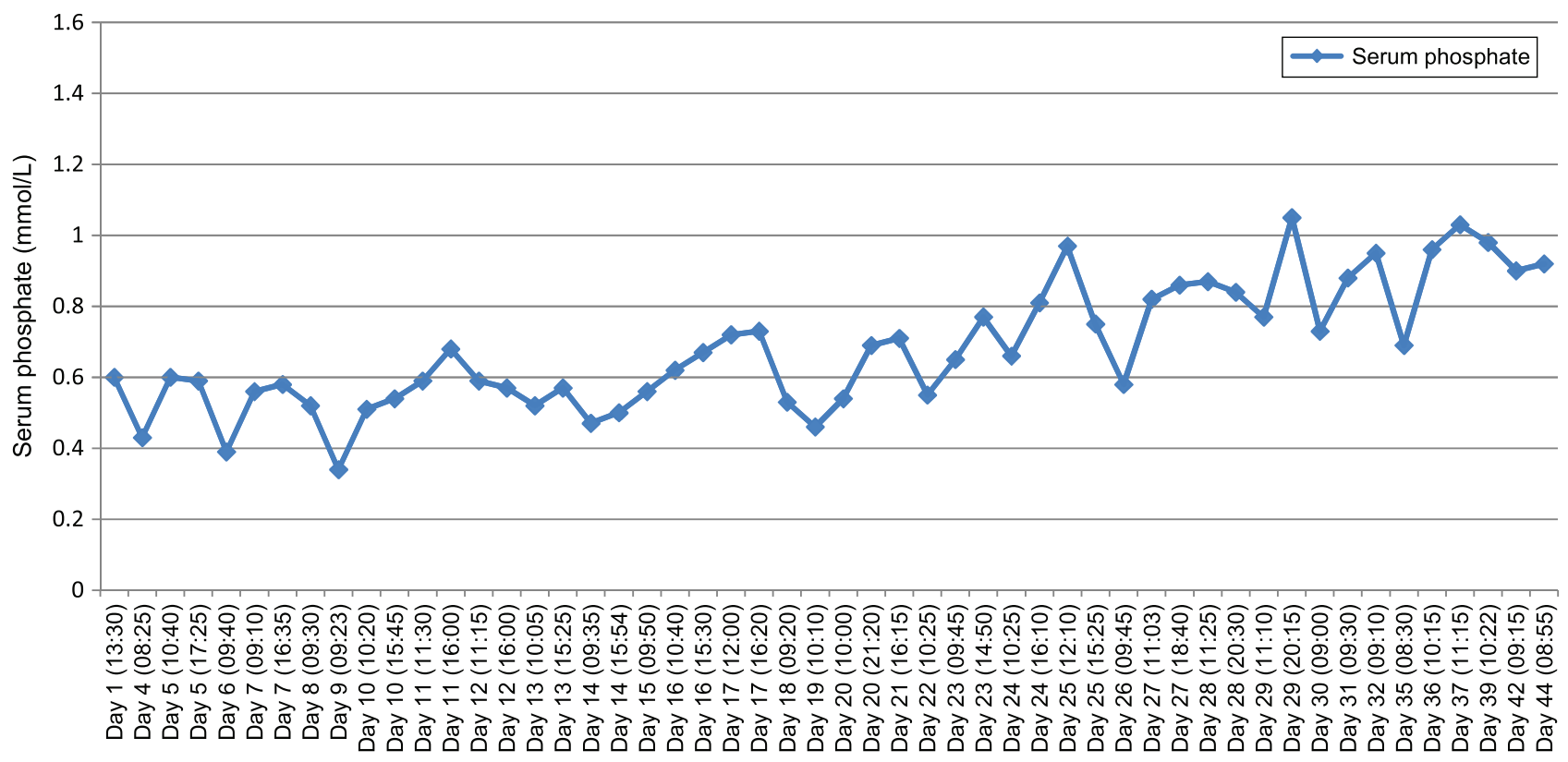

Figure 1

Graph demonstrates the serum phosphate levels at presentation, during admission and after discharge. The sodium dihydrogen phosphate syrup was started on day 17. The last dose of intravenous phosphate therapy was administered on day 26. Patient was discharged home on day 30 . The phosphate supplements were stopped on day 42 . 
thought $(3,4,5)$. The prevalence of hypophosphataemia after high-dose intravenous iron therapy is not easy to ascertain. A study reported that $51 \%$ of patients who received an infusion of ferric carboxymaltose developed hypophosphataemia, the severity of hypophosphataemia correlated with the dose administered, and the mean hypophosphataemia duration was 6 months (6). The main symptom in this group of patients was fatigue.

Despite several case reports in the literature and the fact that this side effect is common, patients are not routinely warned about this potentially life-threatening adverse event before having their iron infusion. Incident reporting of any side effects of medicines to a designated body, even if previously known, is important so that the Medicines and Healthcare products Regulatory Agency (MHRA) or its equivalent can act, if necessary, to ensure that medicines are used in a way that minimises risk, while maximising patient benefit (7). This incident was reported appropriately.

The mechanism by which intravenous iron increases serum FGF23 levels is still not fully understood. Increased production and reduced degradation through various processes have been implicated (8). The carbohydrate moieties in some of the iron preparations (e.g., carboxymaltose in Ferinject ${ }^{\circledR}$ ) have been implicated in altering the FGF23 protein and thereby inhibiting its degradation (8). There are several intravenous iron formulations available and not all cause hypophosphataemia. However, ferric carboxymaltose was found to be more stable, had a better side effect profile, caused less anaphylactic reactions and was found to be well tolerated when compared to other formulations. Additionally, ferric carboxymaltose was initially developed for rapid, single, high-dose infusion (9). Newer preparations, for example, superparamagnetic iron molecules contained within semi-synthetic carbohydrate cores, have been developed and have been shown to exhibit less hypersensitivity, less anaphylaxis and rarely cause hypophosphataemia when compared to other iron preparations (10). It is important to note that many reactions to intravenous iron are incorrectly labelled as anaphylaxis, which is a potentially lifethreatening allergic reaction that usually develops rapidly and may cause death because of circulatory collapse or bronchospasm, and requires immediate treatment. Many of these reactions are mild, self-limiting hypersensitivity reactions (11).

The patient in this case report only had $519 \mathrm{mg}$ of intravenous iron, which resulted in both symptomatic and severe hypophosphataemia within a week. Previous case reports reported hypophosphataemia occurring after administration of higher doses (1000$3000 \mathrm{mg}$ ) of intravenous iron (3, 4, 5, 6). Pre-existing disorders in phosphate homeostasis, including low vitamin $\mathrm{D}$, phosphate and calcium levels have been suggested as risk factors for developing clinically significant hypophosphataemia after intravenous ferric carboxymaltose administration (3, 4, 5, 6). Therefore, measuring these levels prior to having an iron infusion and repeating the serum phosphate level a week after the infusion should be considered for high-risk patients. However, our patient did not have any of these suggested risk factors (e.g., pre-existing vitamin D deficiency, low calcium or low phosphate levels). The patient's vitamin $\mathrm{D}$ and calcium levels were also normal after the iron infusion, but we still prescribed calcitriol hoping that it will help to increase intestinal phosphate absorption and improve serum phosphate levels. Further studies are needed to assess these suggested risk factors. This case was also challenging in that the patient could not tolerate the usual effervescent phosphate tablets and was just about able to tolerate the bespoke syrup. This patient was always symptomatic once serum phosphate levels fell below $0.6 \mathrm{mmol} / \mathrm{L}$ and required multiple doses of intravenous phosphate to keep up with the renal wastage.

Another common feature to all the case reports and case series is the fact that the serum ferritin levels were all in the supraphysiological range for some duration after the iron infusions before falling to the normal range $(3,4,5,6)$. One could speculate that the supraphysiological serum ferritin levels could be a possible 'inflammatory co-factor or co-culprit' disturbing FGF23 metabolism and whether the dose of the iron infusion needs to be altered in order to not produce the initial supraphysiological rise.

In conclusion, much remains to be understood about the mechanism, prevalence and risk factors for developing hypophosphataemia after intravenous iron therapy. This case illustrates the potential severity of this adverse reaction and the need for prescribers to be aware of this potential and occasionally serious complication and the potential need to monitor pre-treatment vitamin D, phosphate, calcium and parathyroid hormone levels and post-treatment serum phosphate levels in patients who may be susceptible. Further research into this phenomenon is required to determine the true prevalence, mechanism and risk factors for intravenous iron-induced hypophosphataemia. 


\section{Patient's perspective}

I was very distressed by the fact that I was not made aware of this serious side effect of having intravenous iron therapy. I was not told what to expect. I certainly was not expecting to end up in hospital for 4 weeks afterwards, which was distressing for both myself and family.

I also found that not many healthcare professionals knew about this side effect: I got most of the information from searching the Google website. The scary part was reading that the condition could last for a long time. What made things worse was the fact that I could not tolerate the Sandoz Phosphate tablets, so I had to keep having the phosphate through an intravenous drip.

My healthcare team read up on the topic and kept me updated throughout my admission and were finally able to get a syrup formulation for me in the end. The syrup still gave my indigestion symptoms.

Healthcare professionals need to be made more aware of this problem and have a plan in place in case this happens to other people. Introducing a pre-screening test to determine the probability of hypophosphataemia occurring, for example, phosphate and vitamin D levels and post-screening phosphate levels. Other iron formulations need to be produced.

I certainly will not be having any iron infusions again in the future.

\section{Declaration of interest}

The authors declare that there is no conflict of interest that could be perceived as prejudicing the impartiality of thid case report.

\section{Funding}

This work did not receive any specific grant from any funding agency in the public, commercial or not-for-profit sector.

\section{Patient consent}

Written informed consent has been obtained from the patient for publication of the submitted article and any accompanying images.

\section{Author contribution statement}

E Ifie and S O Oyibo wrote the initial draft of the article. E Ifie, S O Oyibo, $\mathrm{H}$ Joshi and $\mathrm{O} O$ Akintade all critically revised the paper and approved the final manuscript for submission. S O Oyibo identified the case and is also the named physician for the patient.

\section{References}

1 Moore RA, Gaskell H, Rose P \& Allan J. Meta-analysis of efficacy and safety of intravenous ferric carboxymaltose (Ferinject) from clinical trial reports and published trial data. BMC Blood Disorders 2011114. (https://doi.org/10.1186/1471-2326-11-4)

2 Shamida T, Mizutani S, Muto T, Yoneya T, Hino R, Takeda S, Takeuchi Y, Fujita T, Fukumoto S \& Yamashita T. Cloning and characterisation of FGF23 as a causative factor of tumor-induced osteomalacia. PNAS 200198 6500-6505. (https://doi.org/10.1073/ pnas.101545198)

3 Blazevic A, Hunze J \& Boots JMM. Severe hypophosphataemia after intravenous iron administration. Netherlands Journal of Medicine 2014 72 49-53.

4 Anand G \& Schmid C. Severe hypophosphataemia after intravenous iron administration. BMJ Case Reports 20172017 bcr2016219160. (https://doi.org/10.1136/bcr-2016-219160)

5 Klein K, Asaad S, Econs M \& Rubin JE. Severe FGF23-based hypophosphataemic osteomalacia due to ferric carboxymaltose administration. BMJ Case Reports 20182018 bcr-2017-222851. (https://doi.org/10.1136/bcr-2017-222851)

6 Hardy S \& Vandemergel X. Intravenous iron administration and hypophosphatemia in clinical practice. International Journal of Rheumatology 20152015 468675. (https://doi. org/10.1155/2015/468675)

7 Anderson C, Krska J, Murphy E, Avery A \& Yellow Card Study Collaboration. The importance of direct patient reporting of suspected adverse drug reactions: a patient perspective. British Journal of Clinical Pharmacology 201172 806-822. (https://doi.org/10.1111/ j.1365-2125.2011.03990.x)

8 Wolf M, Koch TA \& Bregman DB. Effects of iron deficiency anaemia and its treatment on fibroblast growth factor 23 and phosphate homeostasis in women. Journal of Bone and Mineral Research 201328 1793-1803. (https://doi.org/10.1002/jbmr.1923)

9 Barish CF, Koch T, Butcher A, Morris D \& Bregman DB. Safety and efficacy of intravenous ferric carboxymaltose $(750 \mathrm{mg})$ in the treatment of iron deficiency anemia: two randomized, controlled trials. Anemia 20122012 1-9. (https://doi.org/10.1155/2012/172104)

10 Auerbach M, Chertow GM \& Rosner M. Ferumoxytol for the treatment of iron deficiency anemia. Expert Review of Hematology 201811 829-834. (https://doi.org/10.1080/17474086.2018.1518712)

11 Kalra PA \& Bhandari S. Safety of intravenous iron use in chronic kidney disease. Current Opinion in Nephrology and Hypertension 2016 25 529-535. (https://doi.org/10.1097/MNH.0000000000000263) 